\title{
EXTENSIONS OF FEFFERMAN-STEIN MAXIMAL INEQUALITIES
}

\author{
Wenhua GaO AND GUOEN Hu
}

Abstract. Let $\beta_{1}, \ldots, \beta_{m} \in[0, \infty)$ and $\mathscr{M}_{L(\log L)^{\vec{\beta}}}$ be the maximal operator defined by

$$
\mathscr{M}_{L(\log L)^{\vec{\beta}}}\left(f_{1}, \ldots, f_{m}\right)(x)=\sup _{Q \ni x} \prod_{j=1}^{m}\left\|f_{j}\right\|_{L(\log L)^{\beta_{j}, Q}} .
$$

In this paper, we establish the weighted bounds in terms of the $A_{\vec{P}}\left(\mathbb{R}^{m n}\right)$ constant for $\mathscr{M}_{L(\log L)^{\vec{\beta}}}$ from $L^{p_{1}}\left(l^{q_{1}} ; \mathbb{R}^{n}, w_{1}\right) \times \ldots \times L^{p_{m}}\left(l^{q_{m}} ; \mathbb{R}^{n}, w_{m}\right)$ to $L^{p}\left(l^{q} ; \mathbb{R}^{n}, v_{\vec{w}}\right)$, where $p_{1}, \ldots, p_{m}, q_{1}, \ldots, q_{m} \in$ $(1, \infty), 1 / p=1 / p_{1}+\ldots+1 / p_{m}, 1 / q=1 / q_{1}+\ldots+1 / q_{m}$ and $\vec{w}=\left(w_{1}, \ldots, w_{m}\right)$ a multiple $A_{\vec{P}}$ weights. A weak type endpoint inequality for vector-valued operator $\mathscr{M}_{L(\log L)^{\vec{\beta}}}$ is also given.

Mathematics subject classification (2010): 42B15. operator.

Keywords and phrases: Maximal inequality, weighted bound, dyadic grid, Lerner's formula, sparse

\section{REFERENCES}

[1] K. F. ANDERSON AND R. T. John, Weighted inequality for vector-valued maximal functions and singular integrals, Studia Math. 69 (1980), 19-31.

[2] T. A. Bui, J. Conde-Alonso, X. T. Duong And M. Hormozi, A note on weighted bounds for singular integral operators with nonsmooth kernels, Studia Math. 236 (2017), 245-269.

[3] D. CruZ-Uribe, SFO, J. MARTEll And C. PÉrez, Sharp weighted estimates for classical operators, Adv. Math. 229 (2012), 408-441.

[4] W. DAMIÁN, A. K. LERNER AND C. PÉREZ, Sharp weighted bouns for multilinear maximal functions and Calderón-Zygmund operators, J. Fourier Anal. Appl. 21 (2012), 751-765.

[5] C. Fefferman And E. M. Stein, Some maximal inequalities, Amer. J. Math. 93 (1971), 107-115.

[6] L. Grafakos, Modern Fourier analysis, GTM250, 2nd Edition, Springer, New York, 2008.

[7] L. GRAFAKOS AND J. M. MARTELL, Extrapolation of weighted norm inequalities for multivariable operators and applications, J. Geom. Anal. 14 (2004), 19-46.

[8] G. Hu, Weighted vector-valued estimates for a non-standard Calderón-Zygmund operator, Nonlinear Anal. 165 (2017), 143-162.

[9] T. Hytönen, The $A_{2}$ theorem: Remarks and complements, Contemp. Math. 612, Amer. Math. Soc. Providence, RI, 2014, 91-106.

[10] T. HytÖNEn, M. T. LACEY AND C. PÉREZ, Sharp weighted bounds for the q-variation of singular integrals, Bull. Lond. Math. Soc. 45 (2013), 529-540.

[11] T. HytÖNEn AND C. PÉREZ, Sharp weighted bounds involving $A_{\infty}$, Anal. PDE. 6 (2013), 777-818.

[12] A. K. LERNER, A poinwisewise estimate for the local sharp maximal function with applications to singular integrals, Bull. London. Math. Soc. 42 (2010), 843-856.

[13] A. Lerner, S. Ombrosi, C. Pérez, R. H. Torres and R. Trujillo-González, New maximal functions and multiple weights for the multilinear Calderón-Zygmund theorey, Adv. Math. 220 (2009), $1222-1264$.

[14] K. LI, K. Moen AND W. Sun, The sharp weighted bound for multilinear maximal functions and Calderón-Zygmund operators, J. Four. Anal. Appl. 20 (2014), 751-765.

[15] C. Pérez, G. Pradolini, R. H. Torres and R. Trujillo-GonzÁlez, End-point estimates for iterated commutators of multilinear singular integrals, Bull. London Math. Soc. 46 (2010), 26-42. 
[16] M. Rao And Z. Ren, Theory of Orlicz spaces, Monographs and Textbooks in Pure and Applied Mathematics, 146, Marcel Dekker Inc., New York, 1991.

[17] E. M. SteIn, Harmonic Analysis, Real Variable Methods, Orthogonality, and Oscillatory Integrals, Princeton Univ. Press, Princeton, NJ. 1993. 